\title{
Electrical conductance of bolted copper joints for cryogenic applications
}

\author{
F. Blondelle • A. Sultan • E. Collin . \\ H. Godfrin
}

Received: date / Accepted: date

\begin{abstract}
We present the results of electric contact resistance measurements at low temperatures on copper-to-copper bolted joints. Our accurate and systematic data display a rather small dispersion, and may be a useful tool for cryogenic applications like pulse-tubes, dilution refrigerators and nuclear refrigerators.
\end{abstract}

Keywords copper joints $\cdot$ conductance $\cdot$ low temperature $\cdot$ cryogenics

PACS $84.32 . \mathrm{Ff} \cdot 07.20 . \mathrm{Mc} \cdot 72.15 . \mathrm{Eb} \cdot 72.15 . \mathrm{Eb}$

\section{Introduction}

The design of low temperature equipment involves thermally connecting parts without introducing significant temperature gradients under the nominal heat flux. Copper is often used for this purpose due to its good thermal conductivity and low cost [1-4]. Since its low temperature thermal conductivity is due to electron transport limited by impurity scattering, substantial work has been performed to obtain high conductivity copper, developing purification and annealing methods [5-7]. As a result, samples with a very large electronic mean free path are obtained, providing large values of the thermal conductivity at sub-Kelvin temperatures [8-11]. It is desirable to introduce joints in the cryogenic system at different places, either for convenience in the original mounting, or in order to be able to change samples, sample holders, experimental cells or nuclear stages in ultra-low temperature research. The joints introduce contact thermal resistances in series with the thermal links, thus degrading the systems performance. Contact resistances are known to depend

F. Blondelle, A. Sultan, E. Collin, and H. Godfrin

Univ. Grenoble Alpes, Inst NEEL, F-38042 Grenoble, France

CNRS, Inst NEEL, F-38042 Grenoble, France

Tel.: +33476889063

E-mail: henri.godfrin@grenoble.cnrs.fr 
on the geometry, the surface conditions, and the applied pressure. The literature provides typical values for different types of joints, measured in different ways, in different conditions. The dispersion of published results, however, is large. We therefore undertook a systematic study of contact resistances in simple copper-to-copper bolted joints, used in our fast-cooling pulse-tube dilution refrigerator equipped with a small copper nuclear stage. This work was developed in the framework of the EU Microkelvin program (www.microkelvin.eu), with the objective of providing more reliable tools for cryogenic design.

\section{Electrical and thermal conductance: Wiedemann-Franz law}

The thermal conductance of a system is defined as $K=P / \delta T$, the ratio of the heat flux (or power) $\mathrm{P}$ and the resulting temperature difference $\delta T=T_{H}-T_{C}$. For a homogeneous sample of length $L$ and cross section $A, K=\kappa A / L$, where $\kappa$ is the thermal conductivity, a characteristic of the material. The quantity of interest in the heat transport through thermal links and joints connected in series, is the inverse of the thermal conductance, the thermal resistance $R_{t h}$ expressed in units of $\mathrm{K} / \mathrm{W}$.

The physics of the electronic thermal and electrical conductivities of metals is described in detail in condensed matter books [12]. At room temperature, the collisions with thermal phonons dominate the electron scattering processes, and the corresponding electrical conductance is independent on the impurity concentration. At low temperatures, below about $10 \mathrm{~K}$, the thermal conductance of a metal is proportional to the temperature, while the electrical conductance is constant: the low temperature or "residual" electrical resistance is determined by the collisions of the electron gas with the impurities.

When the temperature difference across the sample is large with respect to the average temperature, a quadratic temperature dependence of the heat flow is obtained by integration due to the linear temperature dependence of $\kappa$ :

$$
T_{H}^{2}-T_{C}^{2}=\frac{2 L / A}{\kappa / T} P
$$

where $\kappa / T$ is a constant.

The electrical resistance of a metallic sample is determined by its resistivity (for copper, $1.6810^{-8} \Omega \mathrm{m}$ at $20 \mathrm{C}$ ) and geometrical factors. At low temperatures, the resistance is determined by the impurity concentration and the same geometrical factors. The ratio $r$ (called RRR, the residual resistivity ratio) of the electrical resistance at room temperature to that at $4.2 \mathrm{~K}$ is therefore a measure of the purity of the sample:

$$
R R R=r=\frac{R(T=20 C)}{R(T=4.2 K)}
$$

According to the Wiedemann-Franz law, for a metal at low temperatures the ratio of the electronic contribution of the thermal conductivity $\kappa$ to the 
electrical conductivity $\sigma$ is proportional to the temperature $T$ and to the Lorentz number $\mathcal{L}$ :

$$
\frac{\kappa}{\sigma}=\mathcal{L} T
$$

where $\mathcal{L}=2.4410^{-8} \mathrm{~W} \Omega / \mathrm{K}^{2}$; the value determined experimentally for copper is $\mathcal{L}=2.210^{-8} \mathrm{~W} \Omega / \mathrm{K}^{2}[12]$.

Although departures from the Wiedemann-Franz law have been observed in special cases, it is verified with reasonable accuracy in the context of copper samples and joints, which also display a metallic behaviour.

Measuring thermal conductances at low temperatures is not an easy task. It is thus common practice in low temperature work to measure the electrical resistance of metallic samples and make use of the Wiedemann-Franz law to estimate their thermal resistance.

$$
R_{t h}=\frac{R}{\mathcal{L} T}
$$

where $\mathrm{R}$ is the electrical resistance of the sample.

When the temperature difference across the sample is large with respect to the temperature, the quadratic expression is found :

$$
T_{H}^{2}-T_{C}^{2}=\frac{2 R P}{\mathcal{L}}
$$

where $\mathrm{P}$ is the heat flux (power) across the sample.

\section{Conductance values required in typical applications}

Pulse-tube cryocoolers are presently used below $4 \mathrm{~K}$ in a large number of cryogenic devices. We have developed (CNRS/Air Liquide) since 1999 pulse-tube based dry dilution refrigerators, with a commercial unit delievered in 2003 [13]. In these machines, it is important that the condensation stage remains at the lowest temperature, i.e., avoiding thermal gradients at the level of the cold head. Since the cooling power is on the order of $1 \mathrm{~W}$, and the temperature increase around $4 \mathrm{~K}$ should be less than $0.1 \mathrm{~K}$, the thermal resistance should be lower than $0.1 \mathrm{~K} / \mathrm{W}$. Using the Wiedemann-Franz relation, the maximum contact electrical resistance is $10 \mathrm{n} \Omega$.

Cooling samples or a nuclear demagnetization stage with a dilution refrigerator is often performed by bolting copper parts to the bottom of the mixing chamber. At very low temperatures, on the order of $10 \mathrm{mK}$, the cooling power is typically $1 \mu \mathrm{W}$. The thermal contact resistance should therefore be lower than $10^{3} \mathrm{~K} / \mathrm{W}$. The corresponding electrical resistance is $200 \mathrm{n} \Omega$.

The cooling power $P$ of a dilution refrigerator is given by the expression $[1,2]$

$$
P=84 \dot{n}_{3} T^{2}
$$

where the ${ }^{3}$ He flow rate $\dot{n}_{3}$ is expressed in moles/sec, $P$ in Watts and $T$ in Kelvin. 
The dilution refrigerator can thus be represented by an equivalent electrical resistor connected to a bath at zero temperature, as seen from expressions (4) and (5):

$$
R=\frac{\mathcal{L}}{168 \dot{n}_{3}}=1.45 \mu \Omega \frac{100 \mu \mathrm{mol} / \mathrm{sec}}{\dot{n}_{3}}
$$

Contact resistances below $100 \mathrm{n} \Omega$ will be sufficient for connecting samples to typical dilution units with flow rates on the order of $200 \mu \mathrm{mol} / \mathrm{sec}$.

Cooling nuclear stages to less than $10 \mathrm{mK}$ using a dilution refrigerator is achieved through metallic thermal links including a heat switch $[1,2]$. The time needed for cooling a copper nuclear stage of about $1 \mathrm{~kg}$ through the thermal link is on the order of one day, it is therefore desirable to reduce the thermal resistance of the link to a value much lower than the effective resistance of the dilution refrigerator, i.e. to about $100 \mathrm{n} \Omega$. Below about $10 \mathrm{mK}$ one must consider the thermal (Kapitza) resistance between the sintered silver heat exchanger and the helium mixture in the mixing chamber. The Kapitza resistance is in series with the metallic links' resistances. The Kapitza resistivity is $\rho_{K}=14 / T^{2}$ in $\mathrm{K}^{2} \mathrm{~m}^{2} / \mathrm{W}$ for the silver powder we use $[1,2,14]$. For $100 \mathrm{~m}^{2}$ of surface area the Kapitza resistance $R_{K}$ is $1.410^{3} \mathrm{~K} / \mathrm{W}$ at $10 \mathrm{mK}$, equivalent to an electrical resistance of $340 \mathrm{n} \Omega$. This effective resistance depends on temperature as $\mathrm{T}^{-2}$, it is the main limitation for cooling nuclear stages to temperatures below $10 \mathrm{mK}$.

At ultralow temperatures, an electrical contact resistance of $100 \mathrm{n} \Omega$ will cause a temperature difference of $40 \mu \mathrm{K}$ at $100 \mu \mathrm{K}$ for a heat leak of $1 \mathrm{nW}$.

Finally, the thermal links themselves may have a non-negligible resistance. For a copper block of $1 \mathrm{~cm}^{2}$ section and $1 \mathrm{~cm}$ length, with a RRR of 100 , the low temperature electrical resistance is low, $17 \mathrm{n} \Omega$, but a wire made of similar copper of $1 \mathrm{~mm}^{2}$ section and $10 \mathrm{~cm}$ length has a low temperature resistance of $17 \mu \Omega$, clearly unacceptable.

We conclude that contact resistances for the devices considerd here should be in the range 10 to $100 \mathrm{n} \Omega$. The most delicate situations are encountered around $4 \mathrm{~K}$ where large power is applied at the level of the pulse-tube.

\section{Experimental details}

\subsection{Copper samples: Residual Resistivity Ratio}

We have used several copper samples, grouped in the following classes (the type of copper and the date of purchase are indicated):

1) Standard electrolytic copper (Cu-a1) plates $(2013)$

2) Standard electrolytic copper (Cu-a1) plates (1977)

3) Standard OHFC (Cu-c1) copper (2013)

4) OFHC $1 \mathrm{~mm}$ copper plates $>99.9 \%$ (1996), Goodfellow CV000747/15

5) OFHC $1 \mathrm{~mm}$ copper plates, as above (annealed in 2013) 
The samples are representative of the copper purchased by low temperature laboratories. We did not use $\mathrm{Cu}-\mathrm{b} 1$ or $\mathrm{Cu}-\mathrm{b} 2$ phosphorous copper, a poor thermal conductivity material often used for manufacturing copper plates.

\subsection{Low resistance measurements}

The resistance measurements were done using a resistance bridge TRMC2, designed in our laboratory and commercialised by Air Liquide. The low-impedance board has an offset of less than $3 \mathrm{n} \Omega$, and the noise is less than $10 \mathrm{n} \Omega$. The bridge has 28 current (square wave) ranges ( $1 \mathrm{nA}$ to $8 \mathrm{~mA}), 9$ voltage ranges $(5$ to $800 \mu \mathrm{V}), 63$ resistance ranges $(625 \mu \Omega$ to $800 \mathrm{k} \Omega)$. The resolution is $0.002 \%$ (16 bits).

Four-wires measurements were performed using a well-shielded cryogenic set-up, first at room temperature, and then at $4.2 \mathrm{~K}$ in a liquid helium Dewar. Averaging the output signal and checking the offset allowed us to measure resistances with a sensitivity better that $10 \mathrm{n} \Omega$. The absolute accuracy of the measurements, after removing a sample and modifying the wiring configuration, is estimated to be better that $50 \mathrm{n} \Omega$ (error bars indicated in the graphs).

\subsection{Residual Resistivity Ratio measurements}

The table 1 gives the values of the RRR (Residual Resistivity Ratio) determined for the samples of section 4.1 .

Table 1 Residual Resistivity Ratio of copper plates

\begin{tabular}{|c|c|c|c|c|}
\hline Stand.(1) 2013 & Stand.(2) 1977 & OFHC Cu-c1 (3) & OFHC (4) & OFHC (5)Ann. \\
\hline 5 & $80-120$ & 50 & 142 & 480 \\
\hline
\end{tabular}

A major surprize was the observation of very low $R R R$ values (in the range 5 to 10 ) for standard copper plates used in mechanical workshops (several samples from different manufacturers). RRRs on the order of 100 are quoted for $\mathrm{Cu}$-a1 copper in Cryogenics books, values which have been verified in our laboratory several times. We also measured copper samples from old $\mathrm{Cu}$-a1 (before 1980) pieces of equipment, obtaining the values 80-120 given in the table. We conclude that the purity of standard commercial $\mathrm{Cu}$-a1 and also $\mathrm{Cu}$-c1 copper available today is, probably because of recycling, not as good as generally believed. It is therefore necessary to check carefully the RRR of copper material to be used in cryogenic construction. 


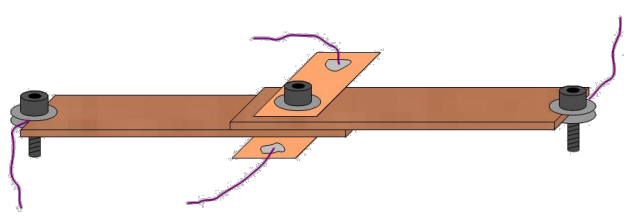

Fig. 1 Schematic drawing of the sample set-up for contact resistance measurements.

\section{Contact resistance measurements}

The set-up shown in figure 1 was used in the measurements of contact resistances. Two copper plates of about $4 \mathrm{~cm}$ length, $1 \mathrm{~cm}$ width and $1 \mathrm{~mm}$ thickness were bolted using stainless steel screws of different diameters, with diffferent steel washers. Current leads were bolted at the opposite ends of the plates, while thin copper ribbons (thickness $70 \mu \mathrm{m}$, width of about $7 \mathrm{~mm}$ ) were placed as shown for the voltage measurement. The measurements were performed at $4.2 \mathrm{~K}$, using the techniques described in section 4.2. The results shown in the following paragraphs, unless stated otherwise, correspond to type 4 samples: OFHC copper plates, $1 \mathrm{~mm}$ thick (see section 4).

\subsection{Bolts, washers, torque and force}

The experiments have been performed using stainless steel hexagonal (Allen) socket head cap bolts, of ISO metric thread M3, M4 or M5. The holes in the copper plates were drilled to the corresponding size (3, 4 and $5 \mathrm{~mm})$, increasing the hole diameter as needed. Stainless steel washers were used: either standard (ISO 7089) flat washers, or Belleville (spring) washers, or both (in this case, the flat washer was in contact with the copper surface).

Table 2 Maximum tightening torque for high quality class stainless steel bolts, resulting forces, and the adequate torque for bolted copper plates.

\begin{tabular}{llll}
\hline Bolt & M3 & M4 & M5 \\
\hline Max. Torque (Nm) & 2.5 & 5.8 & 12 \\
Max. Force (N) & 4100 & 7300 & 12400 \\
Recommended Torque (Nm) & $1-2$ & 2.5 & 3 \\
\hline
\end{tabular}

The bolts were tightened using a torque-limiting wrench. The table 2 gives the maximum tightening torque for very high quality class stainless steel bolts and the corresponding applied force for different diameters. Note that for usual stainless steel bolts the maximum torque is lower by a factor of two (see the data-sheets provided by the manufacturers). The recommended tightening torque determined for our bolted copper contacts (a torque which does not deform permanently the copper or a good quality stainless steel bolt), is also given; it corresponds to forces of about $3000 \mathrm{~N}$. 


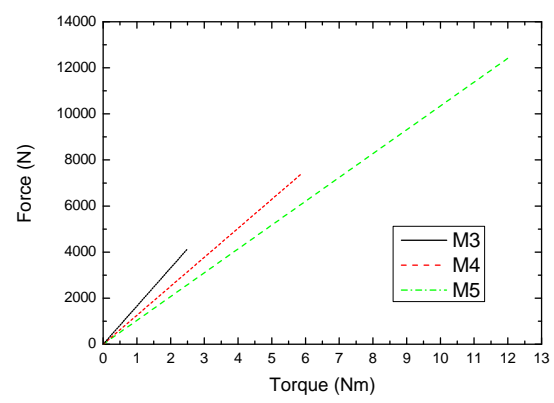

Fig. 2 Force vs. torque relations for different sizes of bolts, drawn up to the limit corresponding to high quality class stainless steel bolts.

The force (fig. 2) is calculated using Kellerman-Klein's expression [15,16]:

$$
T=\left(p / 2 \pi+0,583 d \mu_{t}+D \mu_{h}\right) F 10^{-3}
$$

where $T$ is the applied torque in $\mathrm{Nm}, p$ the pitch in $\mathrm{mm} / \mathrm{turn}, d$ the average diameter in $\mathrm{mm}$ at the bolt flanks, $D$ the average diameter in $\mathrm{mm}$ under the bolt head, $\mu_{t}$ and $\mu_{h}$ the friction coefficients at the threads and under the head, respectively, and $F$ the force in $\mathrm{N}$. The force is the important parameter, which determines the contact resistance: increasing the contact area does not reduce the contact resistance. The friction coefficients are assumed to be on the order of 0.12 ; typical values are 0.1 for greased bolts, and 0.15 as received.

\subsection{Influence of the surface conditions}

The measurements have been performed for different surface conditions. The surfaces were flat (plates), they were either cleaned with emery paper (400grit), and/or in a standard workshop acid bath. In some of the measurements, Apiezon $\mathrm{N}$ vacuum grease was applied as a very thin layer, and the excess was removed with the fingers or a cleaning paper.

As seen in figure 3, the cleaning procedure improves the contact, although much less than commonly believed. Adding a thin layer of grease does neither improve, nor degrade the contact substantially. However, we have observed that thick layers of grease can yield much higher values of the resistance and highly irreproducible results (not shown).

\subsection{Influence of the bolt size}

The data shown in figure 4 show that M3 bolts give relatively high contact resistances. Torques exceeding the values given in the table 2 clearly damage the bolt, matting the bolt threads, thus leading to the observed flattening of 


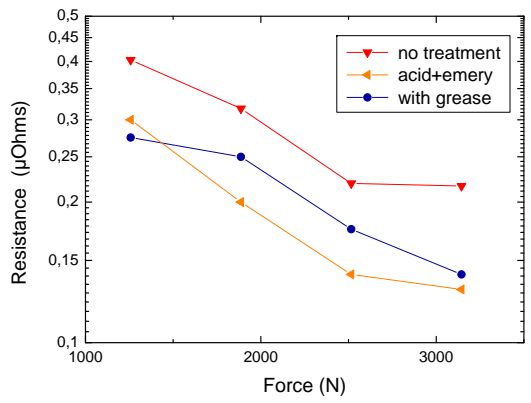

Fig. 3 Influence of the surface conditions on the contact resistance of copper plates bolted using M4 bolts and flat washers. Data: copper plates as received, after cleaning (acid+400grit emery), and with a thin layer of grease.

the resistance vs pressure curve. A substantial improvement is obtained using M4 or M5 bolts. For these, the maximum torque is not determined by the bolt properties, but by the plastic deformation of the copper plates, in particular for annealed samples. The largest applied torques shown in figure 4 correspond to the limit where the washers started imprinting a clear trace on the copper; the contact does not improve further at our highest torque (see figures 4 and 5) and becomes irreproducible. The results obtained with M3 bolts are inconsistent with the assumption that the force is the only relevant physical parameter. One would expect that for small torques,i.e. that do not damage the bolt, the resistance should be comparable to that obtained with larger bolts for the same force. We do not have a quantitative explanation for this effect, but M3 bolts clearly work near their mechanical limit in this context, while M4 bolts work in their optimal range and larger bolts are oversized for this application.

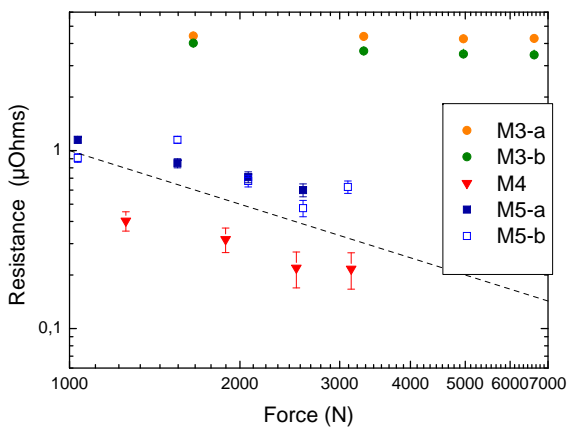

Fig. 4 Influence of the bolt size (M3, M4 or M5; a and b indicate different runs) on the contact resistance of bolted copper plates, using flat washers. The surfaces were cleaned, no grease was used. Dashed line: $1 / \mathrm{F}$ dependence. The force is calculated from the applied torque (see text). 


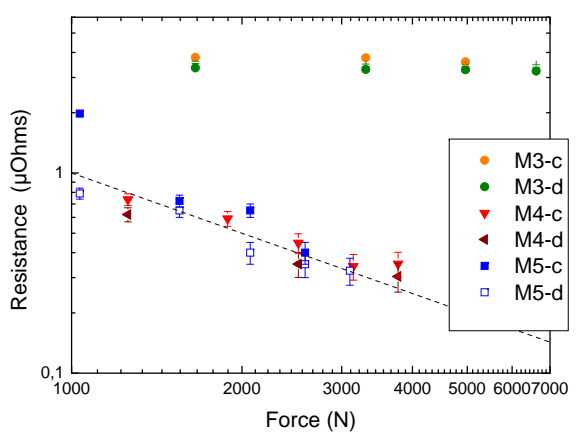

Fig. 5 Influence of grease for different bolt sizes (M3, M4 or M5; c and d indicate different runs) on the contact resistance of bolted copper plates, using flat washers; the surfaces were cleaned; a very small amount of grease was used. Dashed line: 1/F dependence.

As seen comparing figure 4 to figure 5, plating the contacts with a thin layer of Apiezon $\mathrm{N}$ grease does not change significantly the electrical resistance. A thick layer of grease degrades the contact substantially, see section 5.2.

\subsection{Influence of the Belleville washers}

Spring washers (also named Belleville washers) are often used to maintain the pressure when cooling, compensating the effect of thermal contraction of the materials. The results of measurements performed using different combinations of standard (flat) and Belleville washers are shown in figure 6 .

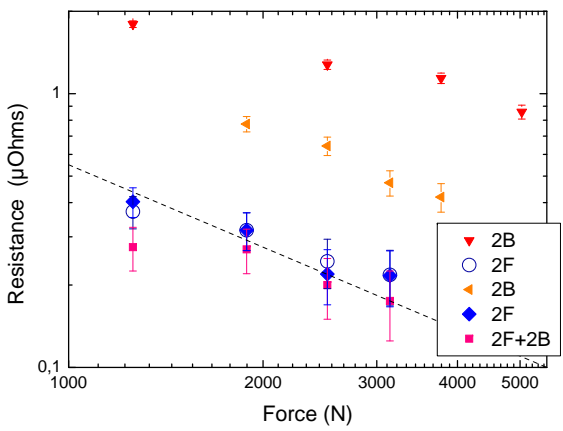

Fig. 6 Contact resistance of copper plates bolted with M4 bolts, using on each side of the sample a flat washer $(2 \mathrm{~F})$, or only a Belleville washer $(2 \mathrm{~B})$, or a Belleville and a flat washer on each side $(2 \mathrm{~F}+2 \mathrm{~B})$, the flat one in contact with the copper. No grease was used. Dashed line: $1 / \mathrm{F}$ dependence. 


\subsection{Influence of other parameters}

The higher resistance data correspond to copper joints with Belleville washers only. In this case, the edge of the conical washers locally exceeds the elastic limit of the copper. As expected, this is not a convenient set-up, but it has the merit of showing clearly the negative effect of localized stress on the quality of copper joints. The measurements display a high irreproducibility (two examples are shown in figure 6). In order to protect the copper and better distribute the pressure, flat washers have been intercalated between the Belleville washers and the copper surfaces (sample $2 \mathrm{~F}+2 \mathrm{~B}$ ). A substantial improvement of the contact is achieved; however, similar results are obtained using only flat washers: the compensation of thermal contraction expected from the Belleville washers, does not play a role.

Other parameters influence the electrical conductance of bolted contacts. Different surface cleaning procedures have been tried. Using 400-grit emery, which creates asperities on the order of $0.4 \mu \mathrm{m}$, seems to be the best method. Sand-blasting the copper parts may seem interesting, but it gave worse contacts. Gold or silver plating improve the contact conductance, and this was verified for some of our samples, silver-plated using commercial solutions. We obtained in this case contact resistances on the order of $50 \mathrm{n} \Omega$ for torques of 2 to $2.5 \mathrm{Nm}$, that is, a factor of 4 better than those obtained for bare copper. Intercalating indium also improved the electrical contact (but indium becomes superconducting at low temperatures). The conductance is larger, but still within the same order of magnitude. We also made measurements using brass bolts; we find that they give contact resistances substantially higher than stainless steel bolts, because lower torques must be used.

\section{Data summary and conclusions}

We present in figure 7 a representative sample of our numerous contact resistance measurements. Our data correspond to copper joints, cleaned in acid and/or with 400-grit emery, bolted using stainless steel M4 bolts and flat washers (we showed that smaller or larger sizes are not particularly useful). The most striking features are the little dispersion of the data for samples of different purity and annealing conditions, and the good proportionality of the electrical conductance to the force. The graph includes results obtained by other groups measuring thermal or electrical resistance. The data in the upper part of the graph correspond to thermal conductance measurements by Berman et al. [17] and Salerno et al. [18-20]. They are clearly well above our data, while following closely, too, a $1 / P$ pressure dependence. In this case, pressure was applied by mechanical devices at low temperatures. Copper demountable joints investigated by Didschuns et al. [21] also displayed the same pressure dependence; they were gold plated, and hence not quantitatively comparable to the samples considered here. 


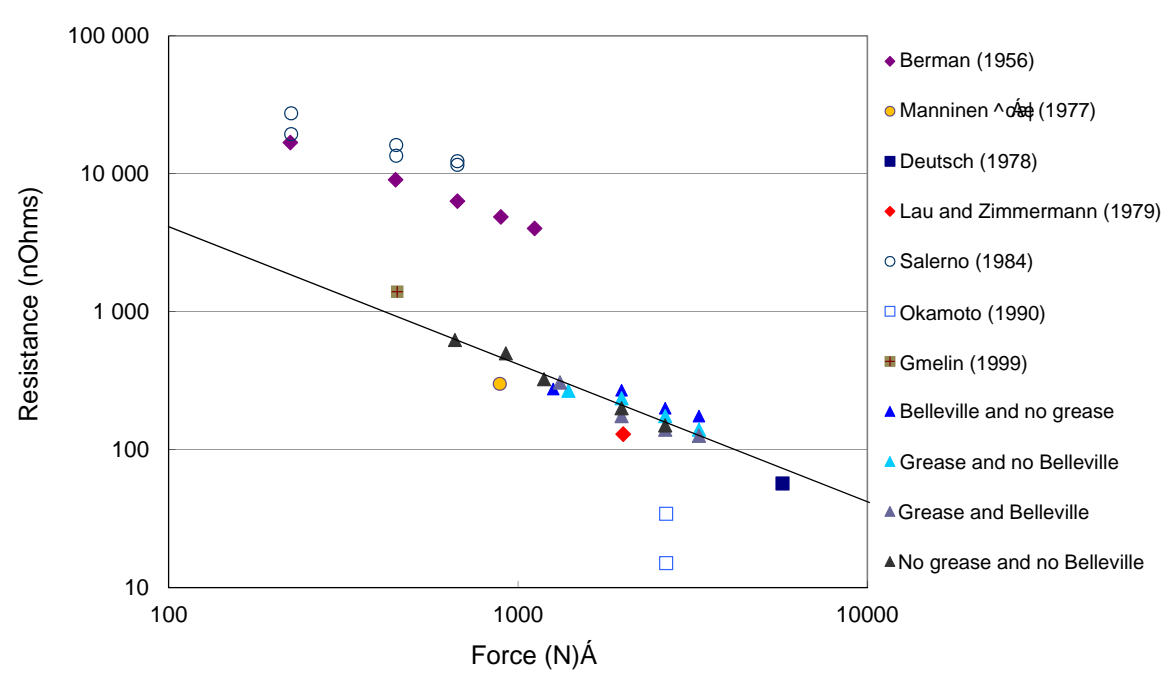

Fig. 7 Summary of the contact resistance results. The upper points correspond to thermal resistance measurements obtained in contacts under mechanical pressure applied at low temperatures; the electrical resistance is deduced using the Wiedemann-Franz law. All other data correspond to electrical resistance measurements. The solid line shows the $1 / F$ dependence obeyed by the data.

Electrical resistance measurements were performed by several groups. The experimental conditions were often different from the simple bolted-plates geometry, but representative results [22-25] have been plotted in figure 7 . Most of the data (for very different geometries) seem to form a coherent set with ours. A notable exception is that of Okamoto et al. [26], who obtain resistance values an order of magnitude lower, in the $10 \mathrm{n} \Omega$ range. The measuring method (current-decay) is different, but the sample conditions are rather similar, we have no explanation for this discrepancy. It would be desirable to perform a simultaneous measurement of the thermal and electrical conductivity on well characterized samples; this is by no means a trivial task, since both measurements are difficult, and some statistics are certainly needed.

The results presented here have been obtained using plates of thickness on the order of $1 \mathrm{~mm}$, but we also measured several samples in the range 0.070 to $6 \mathrm{~mm}$, obtaining similar results within a factor of two. We expect thicker plates to behave differently, because the differential thermal expansion between the bolts and the copper.

We conclude that joints made out of clean (acid bath and/or 400-grit emery paper) millimeter-thick copper plates, using M4 stainless steel bolts and standard flat washers, eventually coated by a protective very thin layer of Apiezon $\mathrm{N}$ grease, provide a simple thermal contact adequate for low temperature work. The applied force is in the $3000 \mathrm{~N}$ range with a tightening torque of about $2.5 \mathrm{Nm}$ (lower than $2 \mathrm{Nm}$ for highly annealed copper samples), sufficient to provide a good contact without damaging the copper parts. The resulting 
contact resistance is on the order of $200 \mathrm{n} \Omega$. This value can be reduced by a factor on the order of 4 , by gold or silver plating. Using several bolted contacts in parallel (optimizing the distance between bolts may be necessary) will be sufficient for many cryogenic applications.

Acknowledgements This work was supported by the European Community Research Infrastructures under the FP7 Capacities Specific Programme, MICROKELVIN project number 228464, and by the grant High-Q Fermions (ANR-2010-INTB-403-01).

\section{References}

1. O. V. Lounasmaa, Experimental principles and methods below $1 \mathrm{~K}$ (Academic Press, London and New York, 1974).

2. F. Pobell, Matter and methods at low temperatures (Springer, Berlin, 2007).

3. R.C. Richardson and E.N. Smith, Experimental techniques in condensed matter physics at low temperatures (Addison-Wesley Pub. Co., New York, 1988).

4. T. Flynn, Cryogenic engineering, 2nd ed. (New York, Marcel Dekker, 2005).

5. D.L. Martin, Rev. Sci. Instr. 38, 1738 (1967).

6. S.S. Rosenblum, W.A. Steyert, F.R. Fickett, Cryogenics 17, 645-647 (1977)

7. W.A. Steyert, J. Appl. Phys. 49, 3612 (1978).

8. C. Y. Ho. R. W. Powell, and P.E. Liley, Thermal Conductivity of the Elements: A Comprehensive Review, supplement no. 1, Journal of Physical and Chemical Reference Data, 3, 1-244,(1974).

9. Cryogenic Properties of Copper, information compiled for the International Copper Association, Ltd. by C.A. Thompson, W. M. Manganaro and F.R. Fickett of the National Institute of Standards and Technology (NIST, Boulder, Colorado, July 1990); http://www.copper.org/resources/properties/cryogenic/

10. J. G. Hust and A. B. Lankford, "Thermal conductivity of aluminium, copper, iron and tungsten from $1 \mathrm{~K}$ to the melting point", NBSIR 84-3007, National Bureau of Standards (Boulder, Colorado, 1984).

11. Copper and dilute copper alloy thermal conductivity calculator, Adam Woodcraft, http://reference.lowtemp.org/

12. C. Kittel, Introduction to Solid State Physics (John Wiley \& Sons Inc., New York, 2005).

13. T. Prouvé, H. Godfrin, C. Gianèse, S. Triqueneaux, A. Ravex, J. Low Temp. Phys. 148, 909 (2007).

14. H. Franco, J. Bossy, H. Godfrin, Cryogenics 24, 477 (1984).

15. R. Kellermann and H.-C. Klein, Untersuchungen ber den Einfluss der Reibung auf Vorspannung und Anzugsmoment von Schraubenverbindungen (Springer Verlag, Berlin, vol. 2, 1955).

16. J. Guillot, Modélisation et calcul des assemblages vissés, Edition Techniques de l'Ingénieur, 2007).

17. R. Berman, J. Appl. Phys. 27, 3183 (1956).

18. L. J. Salerno, P. Kittel, A. L. Spivak, AIAA Journal 22, 1810 (1984).

19. L. J. Salerno, P. Kittel, A. L. Spivak, Cryogenics 33, 1104 (1993).

20. L. J. Salerno, P. Kittel, A. L. Spivak, Cryogenics 34, 649 (1994).

21. I. Didschuns, A. L. Woodcraft, D. Bintley, P. C. Hargrave, Cryogenics 44, 293 (2004).

22. M. Deutsch, Cryogenics 19, 273 (1977).

23. E. Gmelin, M. Asen-Palmer, M. Reuther, R. Villar, J. Phys. D: Appl. Phys. 32 R19

(1999). Review article, see references therein.

24. K. M. Lau, W. Zimmermann, Rev. Sci. Instrum. 50, 254 (1979).

25. M. Manninen, W. Zimmermann, Rev. Sci. Instrum. 48, 1710 (1977).

26. T. Okamoto, H. Fukuyama, H. Ishimoto, S. Ogawa, Rev. Sci. Instrum. 61, 1332 (1990). 